\title{
EXTREME POINTS OF UNIVALENT FUNCTIONS WITH TWO FIXED POINTS
}

\author{
BY
}

\section{HERB SILVERMAN}

ABSTRACT. Univalent functions of the form $f(z)=a_{1} z-\Sigma_{n=2}^{\infty} a_{n} z^{n}$, where $a_{n}>0$, are considered. We examine the subclasses for which $f\left(z_{0}\right)=z_{0}$ or $f^{\prime}\left(z_{0}\right)=1, z_{0}$ real. The extreme points of these classes that are starlike of order $\alpha$ are determined.

1. Introduction. In [7], Schild examined the class of polynomials of the form $f(z)=z-\sum_{n=2}^{N} a_{n} z^{n}$, where $a_{n} \geqslant 0$ and $f(z)$ is univalent in the disk $|z|<$ 1. In [5], Piłat studied the class of univalent polynomials of the form $f(z)=$ $a_{1} z-\sum_{n=2}^{N} a_{n} z^{n}$, where $a_{n} \geqslant 0$ and $f\left(z_{0}\right)=z_{0}>0$. This paper deals with functions of the form

$$
f(z)=a_{1} z-\sum_{n=2}^{\infty} a_{n} z^{n}
$$

where either

$$
a_{n} \geqslant 0, \quad f\left(z_{0}\right)=z_{0} \quad\left(-1<z_{0}<1 ; z_{0} \neq 0\right) .
$$

or

$$
a_{n} \geqslant 0, \quad f^{\prime}\left(z_{0}\right)=1 \quad\left(-1<z_{0}<1\right)
$$

As special cases, some of our results reduce to those of Schild or Piłat. A function $f(z)$ is said to be starlike of order $\alpha, 0 \leqslant \alpha<1$, if

$$
\operatorname{Re}\left\{z f^{\prime}(z) / f(z)\right\}>\alpha \quad(|z|<1)
$$

and is said to be convex of order $\alpha$ if

$$
\operatorname{Re}\left\{1+z f^{\prime \prime}(z) / f^{\prime}(z)\right\}>\alpha \quad(|z|<1) .
$$

Given $\alpha$ and $z_{0}$ fixed, let $S_{0}^{*}\left(\alpha, z_{0}\right)$ be the subclass of functions starlike of order $\alpha$ that satisfy (2), and $S_{1}^{*}\left(\alpha, z_{0}\right)$ be the subclass of functions starlike of order $\alpha$ that satisfy (3). Also denote by $K_{0}\left(\alpha, z_{0}\right)$ and $K_{1}\left(\alpha, z_{0}\right)$ the subclasses

Presented to the Society, January 24, 1976; received by the editors May 7, 1975. AMS (MOS) subject classifications (1970). Primary 30A36; Secondary 30A32. Key words and phrases. Univalent, starlike, convex, extreme point.

Copyright $\odot$ 1976. Americun Muthemutical Suciety 
of functions convex of order $\alpha$ that satisfy, respectively, (2) and (3).

In $\S 2$, we determine necessary and sufficient conditions for functions to be in these classes. In $\S 3$, we find the extreme points for each of these classes. In $\S 4$, we give a necessary and sufficient condition for a subset $B$ of the real interval $(0,1)$ to have the property that $\bigcup_{z_{\gamma} \in B} S_{0}^{*}\left(\alpha, z_{\gamma}\right), \bigcup_{z_{\gamma} \in B} K_{0}\left(\alpha, z_{\gamma}\right)$, $\bigcup_{z_{\gamma} \in B} S_{1}\left(\alpha, z_{\gamma}\right), \bigcup_{z_{\gamma} \in B} K_{1}\left(\alpha, z_{\gamma}\right)$ each forms a convex family. The extreme points of each of these classes is then determined. Many of the results in this paper reduce to those in [8] in the special case $z_{0}=0$.

I would like to thank Professor Eligiusz Złotkiewicz for some interesting discussions concerning this topic.

2. The main subclasses. In [4] it is shown that a necessary and sufficient condition for functions of the form $f(z)=z-\sum_{n=2}^{\infty} a_{n} z^{n} \quad\left(a_{n} \geqslant 0\right)$ to be starlike of order $\alpha$ is that $\Sigma_{n=2}^{\infty}(n-\alpha) a_{n} \leqslant 1-\alpha$.

The proof of the comparable result for functions of the form (1) is essentially the same. See also [8]. We include the proof for the sake of completeness.

THEOREM 1. A function $f(z)=a_{1} z-\sum_{n=2}^{\infty} a_{n} z^{n}\left(a_{n} \geqslant 0\right)$ is starlike of order $\alpha$ if and only if $\Sigma_{n=2}^{\infty}(n-\alpha) a_{n} \leqslant a_{1}(1-\alpha)$.

Proof. Assume that

$$
\sum_{n=2}^{\infty}(n-\alpha) a_{n} \leqslant a_{1}(1-\alpha)
$$

It suffices to show that the values for $z f^{\prime}(z) / f(z)$ lie in a circle centered at $w=1$ whose radius is $1-\alpha$. We have

$$
\left|\frac{z f^{\prime}(z)}{f(z)}-1\right|=\left|\frac{\sum_{n=2}^{\infty}(n-1) a_{n} z^{n}}{a_{1} z-\sum_{n=2}^{\infty} a_{n} z^{n}}\right|<\frac{\sum_{n=2}^{\infty}(n-1) a_{n}}{a_{1}-\sum_{n=2}^{\infty} a_{n}} .
$$

This last expression is bounded above by $1-\alpha$ if (4) is satisfied.

Conversely, assume that

$$
\operatorname{Re}\left\{\frac{z f^{\prime}(z)}{f(z)}\right\}=\operatorname{Re}\left\{\frac{a_{1} z-\Sigma_{n=2}^{\infty} n a_{n} z^{n}}{a_{1} z-\Sigma_{n=2}^{\infty} a_{n} z^{n}}\right\}>\alpha .
$$

Choose values of $z$ on the real axis so that $z f^{\prime}(z) / f(z)$ is real. Upon clearing the denominator in (5) and letting $z \rightarrow 1$ through real values, we obtain (4).

COROllary. A function $f(z)=a_{1} z-\sum_{n=2}^{\infty} a_{n} z^{n}\left(a_{n}>0\right)$ is convex of order $\alpha$ if and only if $\Sigma_{n=2}^{\infty} n(n-\alpha) a_{n} \leqslant a_{1}(1-\alpha)$.

PROof. It is well known that $f(z)$ is convex of order $\alpha$ if and only if $z f^{\prime}(z)$ is starlike of order $\alpha$. Since $z f^{\prime}(z)=a_{1} z-\sum_{n=2}^{\infty} n a_{n} z^{n}$, we may replace $a_{n}$ with $n a_{n}$ in the theorem. 
By choosing particular values for $a_{1} \neq 0$, we obtain some interesting subclasses.

THEOREM 2. Suppose $a_{n} \geqslant 0$ for every $n$. Then $f(z)=a_{1} z-\sum_{n=2}^{\infty} a_{n} z^{n}$ is in $S_{0}^{*}\left(\alpha, z_{0}\right)$ if and only if $\Sigma_{n=2}^{\infty} a_{n}\left[(n-\alpha) /(1-\alpha)-z_{0}^{n-1}\right] \leqslant 1$.

PROOF. Since $f\left(z_{0}\right) / z_{0}=1=a_{1}-\sum_{n=2}^{\infty} a_{n} z_{0}^{n-1}$, the result follows upon substituting

$$
a_{1}=1+\sum_{n=2}^{\infty} a_{n} z_{0}^{n-1}
$$

into the statement of Theorem 1.

REMARK. With a slight modification of the proof of Theorem 3 in [8], we can show under the conditions of Theorem 2 that $f(z)$ is univalent if and only if $\Sigma_{n=2}^{\infty} a_{n}\left(n-z_{0}^{n-1}\right) \leqslant 1$. Hence all such functions are univalent if and only if they are starlike.

COROLlaRY 1. If $f(z)=a_{1} z-\Sigma_{n=2}^{\infty} a_{n} z^{n}$ is in $S_{0}^{*}\left(\alpha, z_{0}\right)$, then

$$
a_{n} \leqslant(1-\alpha) /\left((n-\alpha)-(1-\alpha) z_{0}^{n-1}\right) \quad(n=2,3, \ldots)
$$

with equality for $f(z)=\left((n-\alpha) z-(1-\alpha) z^{n}\right) /\left((n-\alpha)-(1-\alpha) z_{0}^{n-1}\right)$.

COROLLARY 2. Suppose $a_{n} \geqslant 0$ for every $n$. Then $f(z)=a_{1} z-\sum_{n=2}^{\infty} a_{n} z^{n}$ is in $K_{0}\left(\alpha, z_{0}\right)$ if and only if $\Sigma_{n=2}^{\infty} a_{n}\left[n(n-\alpha) /(1-\alpha)-z_{0}^{n-1}\right] \leqslant 1$.

Proof. This follows from the corollary to Theorem 1 as Theorem 2 followed from Theorem 1.

We now examine relationships between the classes $S_{0}^{*}\left(\alpha, z_{0}\right)$ and $K_{0}\left(\alpha, z_{0}\right)$.

THEOREM 3. If $f(z) \in K_{0}\left(\alpha, z_{0}\right)$, then $f(z) \in S_{0}^{*}\left(2 /(3-\alpha), z_{0}\right)$.

Proof. If $f(z) \in K_{0}\left(\alpha, z_{0}\right)$, then $\sum_{n=2}^{\infty} a_{n}\left[n(n-\alpha) /(1-\alpha)-z_{0}^{n-1}\right]$ $\leqslant 1$. In view of Theorem 2 , we wish to find the largest $\beta$ for which $\sum_{n=2}^{\infty} a_{n}\left[(n-\beta) /(1-\beta)-z_{0}^{n-1}\right] \leqslant 1$. It suffices to find the range of values of $\beta$ for which $(n-\beta) /(1-\beta) \leqslant n(n-\alpha) /(1-\alpha)$ for every $n$. Solving, we find $\beta \leqslant$ $2 /(3-\alpha)$. This result is sharp, with extremal function

$$
f(z)=\frac{2(2-\alpha) z-(1-\alpha) z^{2}}{2(2-\alpha)-(1-\alpha) z_{0}}
$$

REMARK. The conclusion of Theorem 3 is true even if $\alpha$ is negative. Thus if $f(z) \in K_{0}\left(\alpha, z_{0}\right)$ for any real $\alpha \leqslant 1$, then $f(z)$ is also starlike and univalent. Note that a function in the class $K(\alpha)$ of normalized univalent convex functions need not be starlike for $\alpha<0$ and need be univalent (and close-to-convex) only if $\alpha \geqslant-1 / 2$. This latter statement follows from a criterion of Kaplan [3], because 
for $z=\mathrm{re}^{i \theta}, 0 \leqslant \theta_{1}<\theta_{2} \leqslant 2 \pi$,

$$
\int_{\theta_{1}}^{\theta_{2}} \operatorname{Re}\left\{1+\frac{z f^{n}}{f^{\prime}}\right\} d \theta \geqslant \int_{\theta_{1}}^{\theta_{2}}-\frac{1}{2} d \theta \geqslant-\pi \quad(\alpha>-1 / 2) .
$$

Observe that $f(z)=\int_{0}^{z}(1-t)^{2(\alpha-1)} d t$ is in $K(\alpha)$ but, by a lemma of Royster [6], is not univalent for $\alpha<-1 / 2$.

THEOREM 4. If $f(z) \in S_{0}^{*}\left(\alpha, z_{0}\right)$, then $f(z)$ is convex in the disk

$$
|z|<r=r(\alpha)=\inf _{n}\left[\frac{n-\alpha}{n^{2}(1-\alpha)}\right]^{1 /(n-1)} \quad(n=2,3, \ldots) .
$$

The result is sharp, with the extremal function being of the form

$$
f_{n}(z)=\frac{(n-\alpha) z-(1-\alpha) z^{n}}{(n-\alpha)-(1-\alpha) z_{0}^{n-1}} \quad(n=2,3, \ldots) .
$$

Proof. It suffices to show that $\left|z f^{\prime \prime}(z) / f^{\prime}(z)\right| \leqslant 1$ for $|z| \leqslant r(\alpha)$. We have

$$
\left|\frac{z f^{\prime \prime}(z)}{f^{\prime}(z)}\right| \leqslant \frac{\sum_{n=2}^{\infty} n(n-1) a_{n}|z|^{n-1}}{a_{1}-\Sigma_{n=2}^{\infty} n a_{n}|z|^{n-1}}
$$

Thus $\left|z f^{\prime \prime}(z) / f^{\prime}(z)\right| \leqslant 1$ if

$$
\sum_{n=2}^{\infty} n(n-1) a_{n}|z|^{n-1}<1+\sum_{n=2}^{\infty} a_{n} z_{0}^{n-1}-\sum_{n=2}^{\infty} n a_{n}|z|^{n-1},
$$

or

$$
\sum_{n=2}^{\infty} a_{n}\left(n^{2}|z|^{n-1}-z_{0}^{n-1}\right) \leqslant 1 .
$$

According to Theorem 2, $\sum_{n=2}^{\infty} a_{n}\left((n-\alpha) /(1-\alpha)-z_{0}^{n-1}\right) \leqslant 1$. Hence (7) will be true if

$$
n^{2}|z|^{n-1}-z_{0}^{n-1} \leqslant(n-\alpha) /(1-\alpha)-z_{0}^{n-1} \quad(n=2,3, \ldots) .
$$

Solving (8) for $|z|$ we obtain

$$
|z| \leqslant\left[(n-\alpha) / n^{2}(1-\alpha)\right]^{1 /(n-1)} \quad(n=2,3, \ldots),
$$

and the result follows.

REMARK. The conclusions in Theorems 3 and 4 are independent of the fixed point $z_{0}$. In [8], these results were proved for the special case $z_{0}=0$.

We turn next to the classes $S_{1}^{*}\left(\alpha, z_{0}\right)$ and $K_{1}\left(\alpha, z_{0}\right)$. If $f(z)$ has the form (1) with condition (3) satisfied, then $a_{1}$ may be expressed as

$$
a_{1}=1+\sum_{n=2}^{\infty} n a_{n} z_{0}^{n-1}
$$


Upon substituting (9) into the statement of Theorem 1 and its corollary, we obtain respectively

THEOREM 5. Suppose $a_{n} \geqslant 0$ for every $n$. Then $f(z)=a_{1} z-\sum_{n=2}^{\infty} a_{n} z^{n}$ is in $S_{1}^{*}\left(\alpha, z_{0}\right)$ if and only if $\Sigma_{n=2}^{\infty} a_{n}\left[(n-\alpha) /(1-\alpha)-n z_{0}^{n-1}\right] \leqslant 1$.

COROLlaRY. Suppose $a_{n} \geqslant 0$ for every $n$. Then $f(z)=a_{1} z-\sum_{n=2}^{\infty} a_{n} z^{n}$ is in $K_{1}\left(\alpha, z_{0}\right)$ if and only if $\Sigma_{n=2}^{\infty} a_{n}\left[n(n-\alpha) /(1-\alpha)-n z_{0}^{n-1}\right] \leqslant 1$.

3. Extreme points. There have been numerous papers recently dealing with the extreme points for the closed convex hull of several compact families of univalent functions. See for example [1] and [2]. The importance of determining the extreme points of a compact family $F$ lies in the fact that the maximum or minimum value of any continuous linear functional defined over the set. of analytic functions occurs at one of the extreme points of the closed convex hull of $F$. Unlike the classes most often considered, $S_{0}^{*}\left(\alpha, z_{0}\right)$ is a convex family. For if $f_{1}(z)$ and $f_{2}(z)$ are in $S_{0}^{*}\left(\alpha, z_{0}\right)$, then $f(z)=\lambda f_{1}(z)+(1-\lambda) f_{2}(z)$ can be shown to satisfy the coefficient inequality of Theorem 2 and $f\left(z_{0}\right)=\lambda f_{1}\left(z_{0}\right)+$ $(1-\lambda) f_{2}\left(z_{0}\right)=z_{0}$. We will now show that the extreme points of $S_{0}^{*}\left(\alpha, z_{0}\right)$ are

$$
z \text { and } z\left[\frac{(n-\alpha)-(1-\alpha) z^{n-1}}{(n-\alpha)-(1-\alpha) z_{0}^{n-1}}\right] \quad(n=2,3,4, \ldots)
$$

TheOREM 6. Set

$$
f_{1}(z)=z \text { and } f_{n}(z)=\frac{(n-\alpha) z-(1-\alpha) z^{n}}{(n-\alpha)-(1-\alpha) z_{0}^{n-1}} \quad(n=2,3, \ldots) .
$$

Then $f(z) \in S_{0}^{*}\left(\alpha, z_{0}\right)$ if and only if it can be expressed in the form $f(z)=$ $\Sigma_{n=1}^{\infty} \lambda_{n} f_{n}(z)$, where $\lambda_{n} \geqslant 0$ and $\Sigma_{n=1}^{\infty} \lambda_{n}=1$.

PROOF. Suppose $f(z)=\Sigma_{n=1}^{\infty} \lambda_{n} f_{n}(z)$, where $\lambda_{n} \geqslant 0$ and $\Sigma_{n=1}^{\infty} \lambda_{n}=1$. Then

$$
f(z)=\left[\lambda_{1}+\sum_{n=2}^{\infty} \frac{\lambda_{n}(n-\alpha)}{(n-\alpha)-(1-\alpha) z_{0}^{n-1}}\right] z-\sum_{n=2}^{\infty} \frac{\lambda_{n}(1-\alpha) z^{n}}{(n-\alpha)-(1-\alpha) z_{0}^{n-1}}
$$

Note that $f\left(z_{0}\right)=\left[\Sigma_{n=1}^{\infty} \lambda_{n}\right] z_{0}=z_{0}$. We also have

$$
\sum_{n=2}^{\infty} \frac{\lambda_{n}(1-\alpha)}{(n-\alpha)-(1-\alpha) z_{0}^{n-1}}\left[\frac{(n-\alpha)-(1-\alpha) z_{0}^{n-1}}{1-\alpha}\right]=\sum_{n=2}^{\infty} \lambda_{n}=1-\lambda_{1} \leqslant 1 .
$$

Hence by Theorem $2, f(z) \in S_{0}^{*}\left(\alpha, z_{0}\right)$.

Conversely, suppose $f(z) \in S_{0}^{*}\left(\alpha, z_{0}\right)$. Since 


$$
a_{n} \leqslant(1-\alpha) /\left((n-\alpha)-(1-\alpha) z_{0}^{n-1}\right) \quad(n=2,3, \ldots),
$$

we may set

$$
\lambda_{n}=\left[\left((n-\alpha)-(1-\alpha) z_{0}^{n-1}\right) /(1-\alpha)\right] a_{n} \quad(n=2,3, \ldots),
$$

and $\lambda_{1}=1-\Sigma_{n=2}^{\infty} \lambda_{n}$. Then $f(z)=\sum_{n=1}^{\infty} \lambda_{n} f_{n}(z)$.

In like manner, the coefficient bounds on $K_{0}\left(\alpha, z_{0}\right), S_{1}^{*}\left(\alpha, z_{0}\right)$, and $K_{1}\left(\alpha, z_{0}\right)$ erable us to prove

THEOREM 7. Set

$$
f_{1}(z)=z \quad \text { and } \quad f_{n}(z)=\frac{n(n-\alpha) z-(1-\alpha) z^{n}}{n(n-\alpha)-(1-\alpha) z_{0}^{n-1}} \quad(n=2,3, \ldots) \text {. }
$$

Then $f(z) \in K_{0}\left(\alpha, z_{0}\right)$ if and only if it can be expressed in the form $f(z)=$ $\Sigma_{n=1}^{\infty} \lambda_{n} f_{n}(z)$, where $\lambda_{n} \geqslant 0$ and $\Sigma_{n=1}^{\infty} \lambda_{n}=1$.

TheOREM 8. Set

$$
f_{1}(z)=z \text { and } f_{n}(z)=\frac{(n-\alpha) z-(1-\alpha) z^{n}}{(n-\alpha)-(1-\alpha) z_{0}^{n-1}} \quad(n=2,3, \ldots)
$$

Then $f(z) \in S_{1}^{*}\left(\alpha, z_{0}\right)$ if and only if it can be expressed in the form $f(z)=$ $\Sigma_{n=1}^{\infty} \lambda_{n} f_{n}(z)$, where $\lambda_{n} \geqslant 0$ and $\Sigma_{n=1}^{\infty} \lambda_{n}=1$.

THEOREM 9. Set

$$
f_{1}(z)=z \quad \text { and } \quad f_{n}(z)=\frac{(n-\alpha) z-((1-\alpha) / n) z^{n}}{(n-\alpha)-(1-\alpha) z_{0}^{n-1}} \quad(n=2,3, \ldots)
$$

Then $f(z) \in K_{1}\left(\alpha, z_{0}\right)$ if and only if it can be expressed in the form $f(z)=$ $\Sigma_{n=1}^{\infty} \lambda_{n} f_{n}(z)$, where $\lambda_{n} \geqslant 0$ and $\Sigma_{n=1}^{\infty} \lambda_{n}=1$.

REMARK. Since the operator $L$ defined by $L f(z)=\int_{0}^{z} f(t) / t d t$ is an isomorphism from $S_{0}^{*}\left(\alpha, z_{0}\right)$ to $K_{1}\left(\alpha, z_{0}\right)$, Theorem 9 is also seen to be a consequence of Theorem 6 .

4. Convex families. Suppose $B$ is a nonempty subset of the real interval $(0,1)$. We define $S_{0}^{*}(\alpha, B)$ by

$$
S_{0}^{*}(\alpha, B)=\bigcup_{\gamma} \in B
$$

As previously mentioned, if $B$ consists of a single element then $S_{0}^{*}(\alpha, B)$ is a convex family. It is of interest to investigate this class for other subsets $B$. We shall make use of the following

LEMMA. If $f(z) \in S_{0}^{*}\left(\alpha, z_{0}\right) \cap S_{0}^{*}\left(\alpha, z_{1}\right)$, where $z_{0}$ and $z_{1}$ are distinct positive numbers, then $f(z)=z$. 
PROOF. Setting $f(z)=a_{1} z-\Sigma_{n=2}^{\infty} a_{n} z^{n}\left(a_{n} \geqslant 0\right)$, we must have

$$
a_{1}=1+\sum_{n=2}^{\infty} a_{n} z_{0}^{n-1}=1+\sum_{n=2}^{\infty} a_{n} z_{1}^{n-1} \text {. }
$$

But this means that $a_{n} \equiv 0$ for $n \geqslant 2$, and the proof is complete.

REMARK. If the condition that the fixed points be positive is relaxed, the conclusion need not follow. For instance if $f(z) \in S_{0}^{*}\left(\alpha, z_{0}\right)$ and $f(z)$ is odd, then $f(z) \in S_{0}^{*}\left(\alpha,-z_{0}\right)$.

THEOREM 10. If $B$ is contained in the interval $(0,1)$ and $0 \leqslant \alpha<1$, then $S_{0}^{*}(\alpha, B)$ is a convex family if and only if $B$ is connected.

Proof. We first assume $B$ is connected. Suppose $z_{0}, z_{1} \in B$ with $z_{0} \leqslant z_{1}$. If $f(z)=a_{1} z-\Sigma_{n=2}^{\infty} a_{n} z^{n}$ is in $S_{0}^{*}\left(\alpha, z_{0}\right), g(z)=b_{1} z-\sum_{n=2}^{\infty} b_{n} z^{n}$ is in $S_{0}^{*}\left(\alpha, z_{1}\right)$, and $0 \leqslant \lambda \leqslant 1$, we will show that there exists a $z_{2}\left(z_{0} \leqslant z_{2} \leqslant z_{1}\right)$ such that $h(z)=\lambda f(z)+(1-\lambda) g(z)$ is in $S_{0}^{*}\left(\alpha, z_{2}\right)$. Set

$$
t(z)=\frac{h(z)}{z}=\lambda a_{1}+(1-\lambda) b_{1}-\lambda \sum_{n=2}^{\infty} a_{n} z^{n-1}-(1-\lambda) \sum_{n=2}^{\infty} b_{n} z^{n-1}
$$

$$
=1+\lambda \sum_{n=2}^{\infty} a_{n}\left(z_{0}^{n-1}-z^{n-1}\right)+(1-\lambda) \sum_{n=2}^{\infty} b_{n}\left(z_{1}^{n-1}-z^{n-1}\right),
$$

and note that $t(z)$ is real when $z$ is real with $t\left(z_{0}\right) \geqslant 1$ and $t\left(z_{1}\right) \leqslant 1$. Hence $t\left(z_{2}\right)=1$ for some $z_{2}, z_{0} \leqslant z_{2} \leqslant z_{1}$. Since $z_{1}, z_{2}$, and $\lambda$ are arbitrary, the family $S_{0}^{*}(\alpha, B)$ is convex.

Conversely if $B$ is not connected we can choose $z_{0}, z_{1} \in B, z_{2} \notin B$, with $z_{0}<z_{2}<z_{1}$. Assume that $f(z)$ and $g(z)$ are not both the identity function. Then, using the notation of (10) except that we fix $z=z_{2}$ and allow $\lambda$ to vary,

$$
t(\lambda)=t\left(z_{2}, \lambda\right)=1+\lambda \sum_{n=2}^{\infty} a_{n}\left(z_{0}^{n-1}-z_{2}^{n-1}\right)+(1-\lambda) \sum_{n=2}^{\infty} b_{n}\left(z_{1}^{n-1}-z_{2}^{n-1}\right)
$$

Since $t\left(z_{2}, 0\right)>1$ and $t\left(z_{2}, 1\right)<1$, there must exist a $\lambda_{0}, 0<\lambda_{0}<1$, for which $t\left(z_{2}, \lambda_{0}\right)=1$. Thus $h(z) \in S_{0}^{*}\left(\alpha, z_{2}\right)$ for $\lambda=\lambda_{0}$. Since $z_{2} \notin B$, an application of the lemma shows that $h(z) \notin S_{0}^{*}(\alpha, B)$. Hence the family $S_{0}^{*}(\alpha, B)$ is not convex.

THEOREM 11. If $\left[z_{0}, z_{1}\right] \subset(0,1)$, then the extreme points of $S_{0}^{*}\left(\alpha,\left[z_{0}, z_{1}\right]\right)$ are $z$,

$$
f_{n}(z)=\left((n-\alpha) z-(1-\alpha) z^{n}\right) /\left((n-\alpha)-(1-\alpha) z_{0}^{n-1}\right) \quad(n=2,3, \ldots),
$$

and

$$
g_{n}(z)=\left((n-\alpha) z-(1-\alpha) z^{n}\right) /\left((n-\alpha)-(1-\alpha) z_{1}^{n-1}\right) \quad(n=2,3, \ldots)
$$


Proof. A function $h(z) \in S_{0}^{*}\left(\alpha, z_{2}\right), z_{0} \leqslant z_{2} \leqslant z_{1}$, can only be an extreme point of $S_{0}^{*}\left(\alpha,\left[z_{0}, z_{1}\right]\right)$ if it is an extreme point of $S_{0}^{*}\left(\alpha, z_{2}\right)$. So we may assume that $h(z)$ is an extreme point of $S_{0}^{*}\left(\alpha, z_{2}\right)$ and show that $h(z)$ is an extreme point of $S_{0}^{*}\left(\alpha,\left[z_{0}, z_{1}\right]\right)$ if and only if $z_{2}=z_{0}$ or $z_{2}=z_{1}$. To show that

$$
h_{n}(z)=\left((n-\alpha) z-(1-\alpha) z^{n}\right) /\left((n-\alpha)-(1-\alpha) z_{2}^{n-1}\right) \quad(n=2,3, \ldots)
$$

can be expressed as a convex linear combination of $f_{n}(z)$ and $g_{n}(z)$ when $z_{0}<$ $z_{2}<z_{1}$, we set

$$
h_{n}(\lambda, z)=\lambda\left(\frac{(n-\alpha) z-(1-\alpha) z^{n}}{(n-\alpha)-(1-\alpha) z_{0}^{n-1}}\right)+(1-\lambda)\left(\frac{(n-\alpha) z-(1-\alpha) z^{n}}{(n-\alpha)-(1-\alpha) z_{1}^{n-1}}\right) \text {. }
$$

For $z$ real and positive, we have $h_{n}(0, z)<h_{n}(z)<h_{n}(1, z)$. Hence there must exist a $\lambda_{0}, 0<\lambda_{0}<1$, for which $h_{n}\left(\lambda_{0}, z\right)=h_{n}(z)$. In fact, for $\lambda_{0}$ such that

$$
\frac{\lambda_{0}}{(n-\alpha)-(1-\alpha) z_{0}^{n-1}}+\frac{1-\lambda_{0}}{(n-\alpha)-(1-\alpha) z_{1}^{n-1}}=\frac{1}{(n-\alpha)-(1-\alpha) z_{2}^{n-1}}
$$

we have the coefficients for $h_{n}\left(\lambda_{0}, z\right)$ agreeing with the coefficients of $h_{n}(z)$. That is, $h_{n}\left(\lambda_{0}, z\right)=h_{n}(z)$ throughout the unit disk when

$$
\lambda_{0}=\frac{(n-\alpha)-(1-\alpha) z_{0}^{n-1}}{(n-\alpha)-(1-\alpha) z_{2}^{n-1}}\left(\frac{z_{1}^{n-1}-z_{2}^{n-1}}{z_{1}^{n-1}-z_{0}^{n-1}}\right)
$$

Thus $h_{n}(z)$ cannot be an extreme point.

On the other hand, we can show for $z$ positive and $0 \leqslant \lambda \leqslant 1$ that

$$
\begin{aligned}
f_{n}(z)<\lambda\left(\frac{(n-\alpha) z-(1-\alpha) z^{n}}{(n-\alpha)-(1-\alpha) z_{3}^{n-1}}\right)+(1-\lambda) & \left(\frac{(n-\alpha) z-(1-\alpha) z^{n}}{(n-\alpha)-(1-\alpha) z_{4}^{n-1}}\right) \\
& \left(z_{0}<z_{3} \leqslant z_{1}, z_{0}<z_{4} \leqslant z_{1}\right)
\end{aligned}
$$

and

$$
\begin{aligned}
g_{n}(z)>\lambda\left(\frac{(n-\alpha) z-(1-\alpha) z^{n}}{(n-\alpha)-(1-\alpha) z_{5}^{n-1}}\right)+(1-\lambda) & \left(\frac{(n-\alpha)-(1-\alpha) z^{n}}{(n-\alpha)-(1-\alpha) z_{6}^{n-1}}\right) \\
& \left(z_{0} \leqslant z_{5}<z_{1}, z_{0} \leqslant z_{6}<z_{1}\right) .
\end{aligned}
$$

This completes the proof.

Using the method of proof in Theorem 11, we obtain the following

COROLlary. If $0<z_{0}<z_{1}<1$, the closed convex hull of $S_{0}^{*}\left(\alpha,\left\{z_{0}, z_{1}\right\}\right)$ is $S_{0}^{*}\left(\alpha,\left[z_{0}, z_{1}\right]\right)$. 
We may similarly expand on the classes $K_{0}\left(\alpha, z_{0}\right), S_{1}^{*}\left(\alpha, z_{0}\right)$, and $K_{1}\left(\alpha, z_{0}\right)$. Our results may be summed up as

THEOREM 12. Let $T\left(\alpha,\left[z_{0}, z_{1}\right]\right), 0<z_{0}<z_{1}<1$, denote any of the classes $S_{0}^{*}\left(\alpha,\left[z_{0}, z_{1}\right]\right), K_{0}\left(\alpha,\left[z_{0}, z_{1}\right]\right), S_{1}^{*}\left(\alpha,\left[z_{0}, z_{1}\right]\right)$, or $K_{1}\left(\alpha,\left[z_{0}, z_{1}\right]\right)$. Then the extreme points of $T\left(\alpha,\left[z_{0}, z_{1}\right]\right)$ are extreme points of $\left.T\left(\alpha, z_{0}\right)\right\} \cup$ \{extreme points of $\left.T\left(\alpha, z_{1}\right)\right\}$, and the closed convex hull of $T\left(\alpha,\left\{z_{0}, z_{1}\right\}\right)$ is $T\left(\alpha,\left[z_{0}, z_{1}\right]\right)$.

\section{REFERENCES}

1. L. Brickman, D. J. Hallenbeck, T. H. MacGregor and D. R. Wilken, Convex hulls and extreme points of families of starlike and convex mappings, Trans. Amer. Math. Soc. 185 (1973), 413-428 (1974). MR 49 \#3102.

2. L. Brickman, T. H. MacGregor and D. R. Wilken, Convex hulls of some classical families of univalent functions, Trans. Amer. Math. Soc. 156 (1971), 91-107. MR 43 \#494.

3. W. Kaplan, Close-to-convex schlicht functions, Michigan Math. J. 1 (1952), 169185 (1953). MR 14, 966.

4. E. P. Merkes, M. S. Robertson and W. T. Scott, On products of starlike functions, Proc. Amer. Math. Soc. 13 (1962), 960-964. MR 26 \#316.

5. Barbara Pirat, Sur une classe de fonctions normées univalentes dans le cercle unité, Ann. Univ. Mariae Curie-Skłodowska Sect. A 17 (1963), 69-73 (1965). MR 33 \#2803.

6. W. C. Royster, On the univalence of a certain integral, Michigan Math. J. 12 (1965), 385-387. MR 32 \#1342.

7. A. Schild, On a class of functions schlicht in the unit circle, Proc. Amer. Math. Soc. 5 (1974), 115-120. MR 15, 694.

8. Herb Silverman, Univalent functions with negative coefficients, Proc. Amer. Math. Soc. 51 (1975), 109-116.

DEPARTMENT OF MATHEMATICS, UNIVERSITY OF DELAWARE, NEWARK, DELAWARE 19711 